\title{
El apocalipsis es decepcionante: el punto muerto despolitizado del consenso sobre el cambio climático
}

\section{Erik Swyngedouw}

Departamento de Geografía. Universidad de Manchester, Reino Unido.

Recibido: 19 de marzo. Aceptado: 11 de agosto.

\begin{abstract}
Publicación original: Pellizzoni, L., Leonardi, E. y Asara, V. (Eds.). Handbook of Critical Environmental Politics. Londres: E. Elgar. En prensa.
\end{abstract}

Traducción: Mónica Farías.

\section{Resumen}

Actualmente existe un consenso generalizado sobre la realidad del cambio climático y la necesidad urgente de tomar medidas inmediatas y de gran alcance. No obstante, a pesar de la preocupación científica y la retórica alarmista, las variables climáticas empeoran. En este artículo sostenemos que la negación de lo Real de los antagonismos políticos y socio-ecológicos que caracterizan el proceso (pos)politizante actual se ve claramente representada en las excentricidades del dilema ecológico en general y, específicamente, en las intervenciones climáticas inefectivas. Sostenemos que el saber sobre el clima se moviliza en un discurso posverdad que oculta aquello que da forma al problema climático y, de este modo, la mirada ambiental queda circunscrita a una cosa fetichizada, asegurando así que nada realmente cambie. Recurriremos a una mirada psicoanalítica lacaniana para salir de este impasse, en particular desde el punto de vista de los activistas y movimientos climáticos más comprometidos. Concluiremos que la base sintomática sobre la que se basa la legitimidad del discurso ambiental, su práctica y la de muchos movimientos climáticos, descansa sobre traumas reprimidos. Desplegar trayectorias político-ecológicas diferentes requiere transgredir la fantasía que oculta estos traumas.

PALABRAS CLAVE: CAMBIO CLIMÁTICO. DESPOLITIZACIÓN. LACAN. POLÍTICAS AMBIENTALES. ACTIVISMO CLIMÁTICO.

\section{The apocalypse is disappointing: the depoliticized deadlock of the climate change consensus}

\begin{abstract}
There is now a widespread consensus about the facts of climate change and the urgent need to take immediate and far-reaching action. Nonetheless, despite the
\end{abstract}


scientific concern and alarmist rhetoric, the climate parameters keep eroding further. In the paper, we argue that the denial of the Real of political and socioecological antagonisms that mark the present (post-)politicizing process is starkly exemplified by the antics of the ecological predicament in general and the impotent climate interventions specifically. We maintain that the knowledge about the climate becomes mobilized in a post-truth discourse that obscures what shapes the climate situation and, in doing so, keeps the environmental gaze circulating around a fetishized thing, assuring that nothing really changes. We shall mobilize a Lacanian psychoanalytically inflected gaze that might elucidate this impasse, in particular from the standpoint of the most passionate climate activists and movements. We shall conclude that the symptomatic base upon which the legitimacy of the environmental discourse, practice and that of many climate movements rests upon repressed traumas. Opening up different political-ecological trajectories requires transgressing the fantasy that conceals these traumas.

KEYWORDS: CLIMATE CHANGE. DEPOLITICIZATION. LACAN. ENVIRONMENTAL POLITICS. CLIMATE ACTIVISM

PALAVRAS-CHAVE: MUDANÇAS CLIMÁTICAS. DESPOLITIZAÇÃO. LACAN. POLÍTICAS AMBIENTAIS. ATIVISMO CLIMÁTICO.

\section{El impasse climático}

Finished, it's finished, nearly finished, it must be nearly finished Endgame (Samuel Beckett, [1957]1958) ${ }^{1}$

En las últimas dos décadas, aproximadamente, se popularizó la cuestión ambiental y, en particular, el cambio climático se convirtió en el núcleo duro de la compleja situación ambiental en la que se encuentra la Tierra. De hecho, el clima ha sido elevado al estatus de preocupación política mundial. Esto fue impulsado por una efectiva alianza entre activistas climáticos cada vez más capaces; las sucesivas reuniones de la Conferencia de las Partes (COP) lideradas por las Naciones Unidas -que mostraron una preocupación aparentemente genuina por parte de la comunidad política internacional-; los trabajos cada vez más alarmistas de la comunidad científica, publicados en la serie de aclamados informes del IPCC, ${ }^{2}$ e intensas campañas mediáticas, por ejemplo, el documental de Al Gore Una verdad inoportuna (Gore, 2006), la cruzada climática del Príncipe Carlos, el documental de Naomi Klein Esto cambia todo (Klein, 2014) o las súplicas apasionadas de Greta Thunberg en pos de medidas para detener el cambio climático. Existe así, en la actualidad, un consenso generalizado sobre la realidad del cambio climático y la necesidad urgente de tomar medidas inmediatas y de gran alcance.

No obstante, a pesar de la preocupación científica y la retórica alarmista, las variables climáticas continúan empeorando. Entre 1990 (año del primer informe del IPCC) y 2018, las emisiones globales de carbono aumentaron un $65 \%$ y continúan aumentando en un $2 \%$ anual aproximadamente. De hecho, la concentración de $\mathrm{CO} 2$ en la atmósfera sigue en ascenso, llegando en la actualidad a más de $400 \mathrm{ppm}$ (partes por millón), el nivel más alto jamás registrado en la historia de la Tierra

1 “Terminó, se terminó, va a terminar, quizá esté por terminar." Final de Partida (Samuel Beckett).

2 Panel Intergubernamental de Expertos sobre el Cambio Climático (nota de la traductora). 
(Observatorio Mauna Loa, 2021). Estos datos demuestran la situación paradójica en la que nos encontramos: confirman el hecho de que la existencia de conocimientos e información, y el acceso a ellos, no garantiza una intervención eficaz. Como lo expresara emblemáticamente el renombrado científico del clima Kevin Anderson (2019:s/p): "A pesar de la retórica optimista, hemos logrado tres décadas de rotundo fracaso en términos de reducción de emisiones totales". "Hasta aquí", continúa, la política climática se ha caracterizado por "una letanía de fraudes tecnocráticos" (Anderson, 2019:s/p). Como parte de esta letanía, incluye, entre otros, "la compensación (... [es como] pagarle a una persona pobre para que haga dieta por nosotros), el Mecanismo de Desarrollo Limpio (CDM) (... compensación sancionada por el Estado), el comercio de emisiones, la forestación (... plantar un árbol, ampliar un aeropuerto) y la geoingeniería (... es como detener una gangrena con una tira adhesiva de tela)", concluyendo con que "iNo hemos tratado de reducir nuestro CO2 seriamente!" (Anderson, 2019:s/p).

En la actualidad, parece inevitable el aumento de la temperatura global de $2 \mathrm{a} 4^{\circ} \mathrm{C}$ para finales de siglo (New et al., 2011). Esto sugiere que muchas de las intervenciones climáticas tienden a ser dispositivos tecno-gerenciales poco efectivos, aunque declarados triunfalmente como capaces de romper con la tendencia evolutiva y estabilizar el clima, de modo que las cosas puedan continuar como de costumbre (para una descripción más detallada, ver Swyngedouw, 2018b).

En este artículo sostenemos que la negación de lo Real de los antagonismos políticos y socio-ecológicos que caracterizan el proceso (pos)politizante actual, se ve claramente representada en las excentricidades del dilema ecológico en general y, en particular, en las intervenciones climáticas inefectivas. Sostenemos que el saber sobre el clima se moviliza en un discurso posverdad que oculta aquello que da forma al problema climático y, de este modo, la mirada ambiental queda circunscrita a una cosa fetichizada asegurando así que nada realmente cambie. Recurriremos a una mirada psicoanalítica lacaniana para salir de este impasse, específicamente desde el punto de vista de los activistas y movimientos climáticos más comprometidos. Esto no pretende ser un argumento en oposición al activismo contra el cambio climático, el cual debe respaldarse por todos los medios posibles. Sin embargo, sí es una afirmación de que gran parte del discurso y la acción en contra del cambio climático reside en una negación posverdad de lo Real de la situación que se deleita con el goce excedente de la actuación histérica, la desesperación melancólica, la acción consumista ética, el apego libidinal al orden socio-ecológico existente y la creencia de que podemos seguir haciendo lo que estamos haciendo, siempre y cuando el $\mathrm{CO} 2$ y sus avatares se gestionen adecuadamente.

Esta conclusión acerca de la impotencia política de la acción ambiental ya fue comentada por Alain Badiou hace más de diez años, cuando polémicamente afirmó sobre la creciente preocupación social y política por la naturaleza, que la problemática ambiental había reemplazado a la religión como el estado de fantasía que confunde lo Real de la situación:

(...) Empecemos por afirmar que después de "los derechos del hombre", el surgimiento de los "derechos de la naturaleza" es una forma contemporánea del opio de los pueblos. Es una religión apenas camuflada... Es una operación gigantesca de despolitización de los sujetos (Feltham, 2008:139). 


\section{Je sais bien, mais quand même... $\mathrm{CO}_{2}$ como objeto-causa del deseo}

A pesar de que se reconoce que el problema del cambio climático es grave, sigue siendo difícil lograr un cambio efectivo. Existe una disonancia cognitiva entre lo que sabemos y cómo actuamos (Dodds, 2011). Como ya lo expresó el teórico psicoanalítico Octave Mannoni en 1969, Je sais bien, mais quand même... (lo sé muy bien [la realidad del cambio climático y la necesidad de una sociedad ecológicamente sensible y socialmente justa], pero aun así [actúo como si no supiera]) (Mannoni, 1969). Para Mannoni (2003), esta es la estructura de la negación fetichista de lo Real de la situación (los mecanismos subyacentes que impulsan el sistema climático), que se presenta como desplazamiento del deseo de un cambio verdadero hacia otra cosa que actúa como reemplazo del deseo mayor e inalcanzable en apariencia. Este espacio vacío es llenado por lo que Jacques Lacan denomina el objeto-causa del deseo u objeto a (Žižek, 1989; Lacan, 2007; Fletcher, 2013; Pohl y Swyngedouw, 2021).

De hecho, el deseo de un futuro justo, ecológicamente benigno y climáticamente estable, en el cual se apoya gran parte de las acciones contra el cambio climático, se desplaza y se articula en torno a la necesidad de reducir las emisiones de gases que producen el efecto invernadero. $\mathrm{El} \mathrm{CO} 2$ y las moléculas asociadas (como el $\mathrm{CH}_{4}$ ) se convierten en la cosa, el objeto causa del deseo -objeto $a$ - alrededor del cual se establece la acción y la simbolización. Sostenemos que lo que constituye la base del actual impasse climático es este desplazamiento fetichista de los procesos socio-ecológicos Reales que producen el cambio climático, hacia un objeto que se considera la causa del problema. Esta negación permite que: 1) el problema del cambio climático pueda tomarse y se tome en serio; 2) la solución se ubique al interior de la transformación/transición tecnológica y de las medidas adaptativas y mitigantes de la gobernanza institucional, de modo que 3) no se tengan que considerar o implementar cambios fundamentales en las actuales relaciones de poder socio-ecológico. Es en este sentido que el debate y la política sobre el cambio climático son en sí mismos despolitizadores, constituyéndose en un nuevo opio de los pueblos (Swyngedouw, 2010b). No serían las relaciones de poder, ya sean de clase u otras, y la expansiva universalidad del capitalismo lo que constituye, las emisiones de gases que producen el efecto invernadero, sino que es esta cosa gaseosa -como configuración en sí misma- alrededor de la cual circula la simbolización y, por lo tanto, las políticas, las intervenciones, los discursos, las instituciones, las acciones y las tecnologías. Esta estructura de fantasía se articula en torno a la creencia de que lidiar con esta cosa es fundamental para la construcción de un mundo diferente y socio-ecológicamente más benigno.

Es esta cuestión espectral imaginaria-material lo que pone en movimiento nuestro deseo, el anhelo de un mundo armonioso, socialmente justo y ecológicamente sensible. Entonces, lidiar con los gases de efecto invernadero ocupa el lugar de la cosa, el hecho imposible -dado que está demasiado fuera de alcance, es demasiado grande para abordarlo, demasiado desconocido, excesivamente atemorizante para contemplarlo, distante hasta lo imposible- que garantizará la satisfacción plena. El CO2 se convierte en un verdadero fetiche. El deseo se transforma así en lo que Lacan llama la pulsión (de muerte), el movimiento incansable e inquieto que circula alrededor del objeto a y que se convierte en "eso" que debe abordarse y alrededor de lo cual circula el goce. Este último es el impulso esquivo (a menudo doloroso y repetitivo) de hacer algo, de actuar en modos que -a menudo sinceramente- creemos reducirán los gases de efecto invernadero (Lacan, 2014). 
El point de capiton, el punto de anclaje por donde pasa la cadena significante que teje la matriz discursiva de significado y contenido de la problemática del cambio climático es, efectivamente, el $\mathrm{CO} 2$. No sólo expresa nuestros miedos más profundos, sino que también hace manifiesto el deseo de cambio. El reduccionismo por duplicado expresado en este componente químico completa la negación fetichista de las relaciones complejas y múltiples a través de las cuales se desenvuelven los cambios ambientales. En primer lugar, las complejas relaciones (de poder) capitalistas socio-ecológicas y la danza histérica de la acumulación de capital que orquesta la emisión de carbono desenfrenada (ver Harvey, 2019) son habitualmente negadas. Al mismo tiempo y, en segundo lugar, se reduce el síndrome patológico a esa cosa objetivada y fetichizada (el $\mathrm{CO} 2 \mathrm{y}$ otros gases de efecto invernadero) que se presenta como el objeto-causa de preocupación, el cual pude controlarse mediante ajustes sociotécnicos, de gestión y de consumo; lo que oscurece aún más las relaciones socio-ecológicas y políticas que sostienen el cambio climático.

La negación de lo Real del cambio climático -lo que no es simbolizable-, mediante el desplazamiento fetichista de sus causas a su presencia sintomática, produce formas de ansiedad neurótica, un sentimiento extraño que aparece cuando la verdad de la situación choca con la impotencia de las acciones realizadas. Esto alimenta comportamientos neuróticos - cuando no algo peor- que a menudo conducen a estados de melancolía, depresión o de distanciamiento cínico (Hoggett, 2013). Hay dos formas de actuación neurótica. El histérico, que es muy consciente de la situación, persiste con la interrogación al Amo (es decir, el orden simbólico existente o el discurso de la modernización ambiental dominante) y le exige que actúe, que cambie de rumbo, que haga lo "correcto". Por otro lado, el activista obsesivo se abocará incansablemente a todo tipo de acciones e intervenciones que reduzcan las emisiones de gases de efecto invernadero. Este tipo de activista cree que el clima y, por lo tanto, el actual orden socio-ecológico colapsarán sin esta actuación obsesiva. Estos dos tipos de impulsos neuróticos dejan intacta la situación actual al tiempo que alimentan y refuerzan el control absoluto de la configuración pospolítica. Fundamentalmente, sostienen la existencia imaginaria de un Amo, al tiempo que señalan hacia aquellos que tienen el poder de actuar y en quienes debemos confiar para saber (Žižek, 1997).

Tal fantasía se sustenta en una doble negación (Weintrobe, 2013). En primer lugar, se insta a los sujetos a actuar: reciclar, reducir la huella de carbono, vivir de manera más sobria, consumir productos socio-ecológicamente "éticos", entre otras acciones. Desde luego existe una extraña y molesta sensación de que en el fondo sabemos que estas respuestas individuales no están a la altura de la tremenda tarea de transformar la dinámica climática: lo sé muy bien, pero aun así... Ciertamente, la complejidad y la naturaleza global del problema indican que las pequeñas acciones individuales son inútiles. Sin embargo, el mandato ético de hacer algo, a pesar de que sabemos que no funcionará, es dominante y a menudo compulsivo. En segundo lugar, las discusiones ambientales le otorgan a la comunidad científica el rol de portadora de la verdad y a las élites políticas el de gobernantes del mundo. En términos lacanianos, se invoca el Discurso de la Universidad (iescuchen a los científicos! ipresionen a los gobernantes!) como medio para cambiar el estado de la situación. Sin embargo, el Discurso de la Universidad es, invariablemente, una estructura en la que se apoya el Discurso del Amo que, efectivamente, sostiene que el medio ambiente presenta un desafío importante al orden existente, pero que insiste en que una combinación de conocimiento (ciencia) 
y control (como la transición sociotécnica y similares) puede mantener al Amo en su lugar (y la confianza en él) (Fink, 1995).

Lo que asegura esto es que las cosas continúen como antes, que podamos mantener un apego libidinal a nuestras vidas tal cual las vivimos, siempre que se implemente la maquinaria climática tecno-institucional apropiada. De esto se desprende la movilización sistemática de significantes vacíos, despolitizantes, que señalan nuestro deseo, pero que a la vez encubren o desplazan lo Real de la situación y que suturan los discursos sobre el cambio climático: "sostenibilidad", "resiliencia", "adaptación", "mitigación”, "transición" (Swyngedouw, 2007). Se trata de significantes sin contenido social o político que, precisamente por eso, a muchos les resuenan, sin que por ello se reconozcan las realidades sociales truncadas y altamente intervenidas que se articulan con la condición socio-ecológica. Es precisamente aquí en donde se torna discernible el vacío o la brecha en el núcleo del discurso climático: son nombres socialmente incorpóreos, consensualizadores, pero vacíos, que ocupan la cadena significante del discurso del cambio climático. Así, niegan radicalmente lo Real de los antagonismos de clase -entre otros antagonismos-, a través de los cuales se constituye tanto la desigualdad como la insostenibilidad. Sin embargo, esta negación de "la verdad" de la situación revela un apego profundamente arraigado al estatus quo, un intento desesperado por asegurar que las cosas continúen como de costumbre.

\section{Sucumbir al populismo climático: la despolitización de la posverdad}

La consensualización establecida sobre el problema del cambio climático, la movilización de un imaginario apocalíptico, la mercantilización tecnocrática de las emisiones de gases de efecto invernadero, la atención puesta en las intervenciones tecno-gerenciales y otras cuestiones similares, se desarrollan en paralelo a la profundización del populismo (Swyngedouw, 2010a; Andreucci, 2019; McCarthy, 2019). El discurso y las políticas climáticas dominantes articuladas en torno a la necesidad urgente de lograr un futuro sensible al cambio climático, debido a la aceleración de una catástrofe de esa índole, se caracterizan, de hecho, por una serie de argumentos populistas y discursos posverdad. Hay paralelos formal-discursivos insólitos entre el populismo xeno-nacionalista de derecha, por un lado, y los argumentos climáticos liberales, presuntamente progresistas, por el otro (ver Canovan, 1999; Mudde, 2004; Laclau, 2005, entre otros). Ambas variantes son síntomas de una despolitización cada vez más profunda del orden socioecológico existente. Podemos resumir en qué consiste la despolitización de este orden climático del siguiente modo:

1. El problema del cambio climático no sólo se presenta como global, sino que además lo hace como una amenaza humanitaria universal. Todos somos víctimas potenciales. El Ambiente y el Pueblo, la Humanidad en su conjunto tanto de manera material como filosófica, están potencialmente en peligro. Sin embargo, el Pueblo no se constituye aquí como un sujeto político heterogéneo, sino como una víctima universal que sufre procesos que escapan a su control. Existe una amenaza externa que es disruptiva y socava una cohesión imaginaria.

Como tal, el argumento atraviesa las idiosincrasias de las "naturalezas" humanas y nohumanas, que son a menudo antagónicas, así como sus "comportamientos" específicos. 
Asimismo, silencia las diferencias ideológicas y otras diferencias sociales y de clase constitutivas, y niega los conflictos democráticos sobre posibles órdenes socio-ecológicos diferentes mediante el enunciado de una amenaza común para la Naturaleza y la Humanidad (Hulme, 2008). Como dice Žižek, "el populismo ocurre cuando una serie de demandas 'democráticas' particulares [en este caso, un buen ambiente, un clima mejorado, una serie de acciones de mitigación socio-ambiental] se encadena en una serie de equivalencias, y este encadenamiento produce 'pueblo' como el sujeto político universal... y todas las diferentes luchas y antagonismos particulares se presentan como parte de una lucha antagónica global entre 'nosotros' (el pueblo) y 'ellos' [en este caso ‘eso', es decir, CO2]”(Žižek, 2017:239).

2. Se refuerza la dicotomía naturaleza-sociedad y el aparente poder causal de la primera para malograr civilizaciones. Neil Smith (2008:245) se refiere a este proceso como "lavado de la naturaleza":

(...) El lavado de la naturaleza es un proceso mediante el cual la naturaleza socialmente modificada se convierte en un super-determinante de nuestro destino como sociedad, a pesar de que está ampliamente aceptado que las transformaciones de la naturaleza son de orden social. Si bien podría ser que el cambio de la naturaleza sea culpa de la sociedad, es el consiguiente poder de la naturaleza la que produce el apocalipsis. El poder causal de la naturaleza no está en riesgo, al contrario, parecería incrementarse mediante las intervenciones sociales en ella (Smith, 2008: 245).

En otras palabras, parecería que al final son los procesos físico-naturales externos, y no las relaciones socio-ecológicas o político-ecológicas que les dan forma, los que requieren un reajuste.

3. Si bien se admite sin reparos que el proceso de acumulación de gases de efecto invernadero es en parte antropogénico, las causas de los problemas ecológicos vinculadas a esto son reconocidas como externas. Así, el $\mathrm{CO} 2$ se convierte en el sustituto fetichizado de todas las calamidades que produce el cambio climático. Por lo tanto, alcanzaría con revertir los niveles de $\mathrm{CO} 2$ atmosférico según un momento histórico que se acuerda como ideal, para volver al estatus quo climático ex ante. De esta forma, se pone en marcha un insólito aparato tecno-gerencial que abarca desde una miríada de nuevas tecnologías ecológicas y propuestas de geoingeniería prometeica, hasta configuraciones gerenciales e institucionales caóticas y complejas que buscan producir una solución socio-ecológica. Consecuentemente, se asegura que no haya cambios fundamentales en el diseño socio-ecológico del mundo (ver, por ejemplo, The Royal Society, 2009; Pellizoni, 2015; Neyrat, 2019). La estabilización del clima se presenta como una condición para que continúe la vida tal cual la conocemos.

4. El enemigo se concibe como un intruso que ha corrompido el sistema. $\mathrm{El} \mathrm{CO} 2$ se presenta aquí como el clásico ejemplo de un enemigo fetichizado y externo que debe ser controlado. Por lo tanto, los problemas no serían el resultado del "sistema", de las relaciones de poder desiguales, de las redes de control e influencia, de las crecientes injusticias o, inclusive, de una falla funesta intrínseca al sistema, sino que serían culpa de un extraño (Žižek, 2006). El discurso consensuado “desplaza el antagonismo social y construye al enemigo... el enemigo es puesto fuera o cosificado como una entidad 
ontológica positiva [CO2 excesivo] (incluso aún si esta entidad es espectral) cuya aniquilación restablecería el equilibrio y la justicia” (Žižek, 2006:555).

5. Esa es la razón por la cual la solución consiste en lidiar con el fenómeno "patológico", el farmakon, en tanto el mismo reside al interior del sistema. El "enemigo" permanece socialmente vacío y se homogeneiza; es una simple cosa, no encarnada socialmente, nombrada o contada. Si bien una política adecuada acompañaría la idea de que el CO2como-crisis se presenta como el síntoma patológico de lo normal (síntoma que expresa los excesos inscritos en el normal funcionamiento del sistema), la arquitectura política dominante en torno al cambio climático insiste en que este estado (el CO2-como-crisis) es ajeno al sistema. Al mismo tiempo, se le asignan cualidades profilácticas a la puesta en acción de la propia dinámica y lógicas internas del sistema que produjo el problema en primer lugar. De ahí el énfasis en las fuerzas del mercado (privatización, mercantilización e intercambio de mercado del, a menudo ficticio, CO2) (Swyngedouw, 2018a).

6. El consenso climático es invocado en el Nombre del Pueblo, pero se apoya en una tecnocracia científica supuestamente neutral que aboga por una relación directa entre las personas y la participación política y que, a menudo sin mucha mediación política, hace de las cuestiones de hecho, cuestiones de interés (Latour, 2004).

Se supone que esto conducirá a una solución que, si no óptima, será al menos buena. La arquitectura del gobierno consensual funciona a través de la participación de las partes interesadas o mediante formas de gobierno participativo que operan más allá del Estado y que, bajo el ala de un orden liberal capitalista incuestionable, nutren formas de autogestión, autoorganización y autodisciplina (Dean, 1999; Swyngedouw, 2005). En estas tácticas consensuales no se identifica a un sujeto de cambio privilegiado (como el proletariado para los marxistas, las mujeres para las feministas o la "clase creativa" para el capitalismo competitivo), sino que se invoca a una condición o a un dilema común: la necesidad de una acción en conjunto de toda la humanidad, la colaboración multiescalar y la cooperación. No hay tensiones sociales internas ni conflictos internos. Sin embargo, es precisamente la escisión constitutiva del pueblo, el reconocimiento de que hay deseos sociales, políticos o ecológicos radicalmente diferenciados, y a menudo opuestos, lo que hace nacer lo político.

7. El problema ecológico, en su formato populista, no propone una transformación del actual orden socio-ecológico, sino que convoca a las élites a actuar de modo que nada tenga realmente que cambiar, de modo que la vida pueda continuar básicamente como antes. En este sentido, el consenso acerca del cambio climático es inherentemente reaccionario, es un andamiaje ideológico (o más bien imaginario) para asegurar el estatus quo sociopolítico. Es inherentemente apolítico y no partidista. En esta línea, durante los últimos años se ha dado una suerte de "revolución pasiva" gramsciana en la que las élites reconocen el problema del cambio climático, respondiendo así al reclamo del "pueblo" de tomarse el clima en serio, pretendiendo además convencer al mundo de que no sólo el capitalismo puede resolver el problema, sino que de hecho este se encuentra en condiciones de producir un clima mejorado y más sustentable al deshacer aquel que ha coproducido durante los últimos cientos de años.

8. La gobernanza climática pospolítica (ver Swyngedouw, 2010a) no resuelve los problemas, sino que los desplaza. Consideremos, por ejemplo, el debate actual en torno a la 
energía nuclear como opción viable y realista para asegurar la sustentabilidad energética y como una alternativa para hacerle frente tanto a las emisiones de $\mathrm{CO} 2$ como al zenit petrolero (aún después del horror de Fukushima). La "solución" nuclear es a menudo presentada como un remedio que puede salvar al clima y al capital, aunque difícilmente genera esperanzas de una sociedad mejor y ecológicamente sana.

9. Las demandas y los proyectos populistas generalmente se dirigen a las "élites", a quienes se las insta a actuar: iDetengan la inmigración! iSalven el Clima! iCuren el Covid-19!... El populismo ecológico es inherentemente no partidista y aparentemente no ideológico y no intenta cambiar la composición de las élites, sino que les pide que utilicen el poder que tienen para actuar.

10. Lo más problemático es que en la política consensual pospolítica no se identifican nombres propios. El populismo pospolítico se asocia a una política que no-nombra, en el sentido de dar un nombre preciso o propio a su campo de acción. Los nombres propios de la política son reemplazados por conceptos imprecisos como política de cambio climático, política de biodiversidad o por un discurso de la sustentabilidad vacío de contenido. Sin embargo, según Jacques Rancière, son los nombres propios los que constituyen una democracia genuina, es decir, un espacio en donde lo innombrado, lo incontable y, por consiguiente, lo no-simbolizado se nombra y se cuenta (Rancière, 1998). El cambio climático no tiene un nombre o un significante político encarnado; no convoca a la existencia de un sujeto político o, más bien, no hay sujeto político que se invista de su nombre. A diferencia de otros significantes que decididamente revelan un contenido encarnado con relación al futuro (como el socialismo, el comunismo o el liberalismo), un mundo futuro, ecológica y climatológicamente diferente, sólo se expresa en su negatividad; una negatividad pura sin promesas de redención, sin un mandamiento positivo que "trascienda" / sublime la negatividad, sin sujeto político. No obstante, la mirada puesta en el mañana hace que se reformulen los problemas sociales, políticos y demás temas urgentes del presente como condiciones del futuro que se reescriben retroactivamente como una cuestión tecno-gerencial. Mientras las demandas se presentan como algo particular (por ejemplo, reducir $\mathrm{CO} 2$ ), la pospolítica impide que la universalización sea parte de un proyecto socioambiental positivo. En otras palabras, el problema ambiental no estaría planteando una situación socioambiental que se pueda nombrar y que sea favorable, ni una mirada encarnada, ni un deseo que espera ser concretado, ni una pasión a realizar.

Pareciera como si este discurso populista sobre el clima ejerciera un atractivo inexorable que en gran parte comparten tanto liberales y progresistas como conservadores eco-modernistas. Esta atracción fantasmagórica amerita mayor atención.

\section{La inmuno-biopolítica de la adaptación y reducción del cambio climático}

Frente a la amenaza de una naturaleza irremediablemente vengativa, los dispositivos sociotécnicos y de gestión de la gobernanza del cambio climático actual prometen y proveen (aunque de modo muy desigual socio-ecológicamente) una profilaxis en apariencia inmunológica. Estas expresiones inmunológicas dirigen nuestra supervivencia hacia el futuro sin considerar la necesidad, o la posibilidad, de una transformación 
de las relaciones socio-naturales; así, se proponen y fomentan las adaptaciones tecno-gerenciales para asegurar la "sustentabilidad" de lo existente, aunque esta sea un significante vacío (Swyngedouw, 2007). En el mejor de los casos, las políticas frente al cambio climático, centradas en acuerdos sociotécnicos y apoyadas en la existencia de mediadores que mitigan la huella eco-física de los humanos (desde el comercio de carbono hasta la fabricación de sumideros de carbono y de fuentes de energía alternativas), pueden sólo proporcionar un paliativo que posponga momentáneamente el Endgame (Final de partida) que termine con una tierra inhabitable. Al mismo tiempo ofrecen, en un formato al estilo de camisa de fuerza eco-modernizadora, la promesa de una reinterpretación radical que, sin embargo, mantiene en su curso al capitalismo. Es decir, para ponerlo en palabras de Roberto Esposito, se promete la elaboración de una biopolítica inmunológica que supuestamente garantizará nuestra supervivencia (Esposito, 2008, 2011, 2012).

Esposito insiste en que la gubernamentalidad neoliberal contemporánea está suturada por un impulso inmunológico que garantiza que la vida continúe (Neyrat, 2010). El mismo tiene como objetivo aislar herméticamente al objeto de gobierno (la población) para protegerlo de la amenaza potencial de intrusos peligrosos y extraños ingobernables -o desestabilizantes- que puedan poner en riesgo la bio-felicidad de la población, cuando no la misma supervivencia. Un ejemplo de esto es la desigual gobernanza biopolítica de la pandemia de Covid-19. En este caso entendemos lo inmunológico como la interrupción de la obligación del intercambio de obsequios en una comunidad. La libertad individual y la libertad de elección como mandato (neo)liberal son, precisamente, el gesto fundante de una biopolítica inmunológica elevada a mandato fundamental de las formas neoliberales de gubernamentalidad. El trabajo de la inmunopolítica se evidencia, por ejemplo, en las prácticas hegemónicas occidentales en torno al ambiente, a la inmigración o al terrorismo internacional. De hecho, un arsenal de tecnologías blandas y duras en rápida expansión es puesto en uso en una capa aún más densa de dispositivos inmunológico-técnicos, de infraestructura y legal-institucionales -que abarcan desde las leyes de inmigración, cada vez más duras y de vigilancia constante, hasta la construcción de muros y barreras de acero y cemento, por no hablar del acordonamiento estricto cuando enfermedades infecciosas amenazan con espacializarse de modo que podrían entrar en las burbujas de inmunidad específicamente diseñadas para la élite local-. El estado inmunológico se ocupa del trabajo sucio para que los objetos de la gubernamentalidad biopolítica continúen la vida cotidiana protegidos en una suerte de cápsulas geopolíticas y geo-ecológicas acapulladas, al menos en apariencia, libres de los peligros y riesgos que atraviesan nuestras ganas de vivir una vida arcádica. Así, los intentos de geo-diseñar el clima a través de una desviación solar masiva, el almacenamiento de carbono a grandes profundidades o la adaptación a gran escala, entre otros ejemplos, prometen una profilaxis inmunológica que permite que la vida continúe sin grandes cambios sociales o político-económicos, aunque sea para algunos (Neyrat, 2014, 2019). Como dice Pierre-Oliver Garcia: "Un poder inmunitario controla los riesgos, los peligros y las debilidades de los individuos para hacerlos vivir de manera pacífica mientras se oculta cualquier forma de disenso" (Garcia, 2015:321).

De hecho, la búsqueda de una biopolítica inmunológica es uno de los pocos terrenos que le quedan al poder estatal en un mundo en donde la privatización-mercantilización de todo, combinada con un vaciamiento de su soberanía territorial -precisamente debido a los procesos de globalización-, ha fomentado la reorientación de las funciones 
estatales hacia la gestión de la población y su felicidad. La inmuno-biopolítica es, por lo tanto, uno de los dispositivos estructurantes de vital importancia en el proceso de hegemonización de la pospolitización tecno-gerencial (Ernstson y Swyngedouw, 2018).

Sin embargo, si bien se logra exitosamente que las estrategias inmuno-biopolíticas con relación a la migración, al terrorismo o al riesgo económico-financiero se desplacen, identificándose no ya con el miedo a la desintegración, sino con un discurso y práctica de gestión de la crisis por parte de las élites políticas, este recurso fracasa cuando se trata de la catástrofe ecológica conjunta y desigual. ¿No es acaso cierto que las técnicas de gestión inmuno-política como la política climática adaptativa, los acuerdos sociotécnicos sostenibles, la mercantilización de los gases de efecto invernadero, los planes climáticos ambiciosos pero ineficaces, etc. generan una constante sensación de malestar e incomodidad frente a variables socio-ecológicas cada vez más deterioradas, mientras que las diversas medidas tomadas ofrecen, en el mejor de los casos, sólo un paliativo temporal? De hecho, son los mismos mecanismos que supuestamente producen una respuesta inmunológica los que amenazan con socavar aún más las condiciones socioecológicas. La inmuno-biopolítica se convierte así en una tanatopolítica o necropolítica de autoinmunidad (Mbembe, 2003).

La destrucción socio-ecológica es un hecho apremiante que socava dramáticamente esta fantasía inmunológica, dejando en evidencia la brecha insalvable entre lo Simbólico (el discurso y la práctica de la actual política climática) y lo Real (el cambio climático acelerado). La extraña sensación, si no la ansiedad, de que las cosas no son realmente como deberían ser resulta irritante, al tiempo que se sublima y objetiva en lo que anteriormente nombramos objeto a: la cosa horrible alrededor de la cual circulan tanto el miedo como el deseo. Los intentos de captura y aislamiento rotundamente fallidos de $\mathrm{CO} 2$ cada vez más requieren de acciones precisas para mantener a raya la catástrofe. En otras palabras, el dispositivo inmunológico demanda que se lo renueve y profundice. Esto es lo que prometen los proyectos y las propuestas de geoingeniería mega-prometeicas, lo que una vez más respalda la fantasía de que se puede posponer la catástrofe climática a una lejana imposibilidad. La fantasía inmunológica, y ciertamente performativa, de que es posible mantener un profundo apego libidinal a la vida individual y social que llevamos (a pesar de las afirmaciones a menudo enérgicas y apasionadas de lo contrario), fuerza al discurso sobre el cambio climático en una dirección despolitizante en donde el debate se circunscribe a discusiones sobre cuál es la tecnología apropiada, la forma institucional adecuada para los acuerdos de gestión y la cantidad de gases de efecto invernadero que se pueden ahorrar o desplazar (Fletcher, 2018).

\section{Transgrediendo la Fantasía: el apocalipsis es decepcionante}

La legitimidad del discurso ambiental y de su práctica, y la de muchos movimientos climáticos, se apoya sobre una base sintomática que descansa en dos traumas reprimidos y desplazados a un imaginario fantasmagórico. Abrirse a trayectorias político-ecológicas diferentes requiere transgredir la fantasía que oculta estos traumas.

En primer lugar, vemos la construcción persistente de un futuro distópico cuasi catastrófico. Esta imagen se presenta como el horizonte a ser evitado o desviado. En otras palabras, se requiere de una acción urgente hoy para evitar que este futuro posible se 
concrete. Por supuesto esta visión alimenta la idea de que aún no es demasiado tarde, que se puede evitar el futuro predicho. Sin embargo, muchas personas alrededor del globo ya viven una catástrofe socio-ecológica, demostrada por el gran número de refugiados climáticos y los crecientes problemas socio-ecológicos en las regiones más pobres del mundo (Parenti, 2011; Miller, 2017). El apocalipsis ya ocurrió para ellos. Al temor a las consecuencias del cambio climático en un lugar, lo acompaña la desintegración socio-ecológica de hecho en otros lugares. Los deseos y los miedos de la clase media circulan alrededor de la promesa de una catástrofe en ciernes. Mientras que las élites promueven una distopía apocalíptica que, a pesar de todo, puede ser evitada (para ellos), la mayoría del mundo ya está viviendo "al interior del colapso de la civilización" (The Invisible Committee, 2009:96). El apocalipsis es, de hecho, conjunto y desigual, tanto en el tiempo como en el espacio (Calder, 2011; Harper, 2020).

Planteamos que una parte fundamental y vital de la nueva política cultural del capitalismo, cuyo leitmotiv central es la gestión del miedo (Badiou, 2007), es promover y sostener imaginarios catastróficos que forman parte del apoyo cultural para un proceso de pospolitización populista. A nivel simbólico, los imaginarios apocalípticos cumplen un rol importante en la negación o desplazamiento del conflicto social y los antagonismos. En otras palabras, la presentación del cambio climático como una causa humanitaria a nivel global produce un imaginario completamente despolitizado en donde no se trata de elegir una trayectoria en lugar de otra o de identificar enemigos dentro de un proceso político. En su lugar, se trata de un imaginario que no se articula con programas políticos específicos, con proyectos o transformaciones socio-ecológicas (Swyngedouw, 2018b).

Al transgredir esta fantasía se llega al núcleo del punto muerto sobre el consenso acerca del cambio climático. Para empezar, se debe rechazar por completo la promesa reveladora de la narrativa apocalíptica. Frente a los imaginarios cataclísmicos puestos en circulación para asegurar que el apocalipsis NO ocurrirá (si se toman las acciones tecno-gerenciales adecuadas), la única respuesta razonable es: no se preocupen (ecomodernizadores, Príncipe Carlos, reuniones de la Conferencia de las Partes, numerosos activistas ambientales.), tienen mucha razón, la catástrofe ambiental no sólo SUCEDERÁ, sino que ya sucedió, YA ESTÁ AQUÍ. Muchos ya están viviendo en los intersticios de la vida post apocalíptica, en donde la combinación del cambio ambiental y de las condiciones sociales hacen que la vida se presente "desnuda". Debe quedar claro que el problema socioambiental ya ha pasado el punto de no retorno. Para muchos, la ruina socioambiental ya llegó; no se trata de una promesa distópica distante puesta en circulación para provocar una respuesta en el presente. Los conflictos por el agua, las luchas por el alimento, los refugiados ambientales, etc. dan testimonio de la difícil situación socio-ecológica que condiciona la vida cotidiana de la mayoría de la población mundial. Ya es tarde; siempre fue tarde. No hay un lugar, un tiempo o entorno arcádico al que se pueda volver, ni un pasado socio-ecológico global benigno, ni un clima ideal que se deba mantener o estabilizar. Una nueva política sólo puede surgir al interior de la conciencia de la realidad apocalíptica actual.

En segundo lugar, el discurso climático se activa al invocar los peligros inmanentes que el cambio climático representa para la humanidad. En este contexto, la humanidad se lee como civilización humana y no sólo como la suma de seres humanos en el planeta Tierra. Como ya argumentara Maurice Blanchot a principios de la década de 
1960, esta mirada se basa, por supuesto, en la fantasía de que la humanidad existe realmente, de que existe una civilización humana global que requiere o merece la salvación. Nuevamente, queda al descubierto el núcleo vacío de la humanidad. El conocimiento de que no existe tal humanidad es, de hecho, un trauma reprimido (Blanchot, 1971). Las disparidades por doquier, las relaciones desiguales de poder que no dejan de crecer, la violencia permanente -tanto objetiva como subjetivaque algunos humanos ejercen sobre otros (considérese, por ejemplo, el genocidio causado por la Unión Europea a los refugiados que mueren en el Mediterráneo o en los campos de concentración del norte de África) ponen de relieve los antagonismos fundamentales y los conflictos que atraviesan lo humano, al tiempo que son un signo de que la humanidad comunitaria nunca existió. Y puede que nunca exista, a menos que haya un compromiso político sostenido en el tiempo sobre la posibilidad de que así sea. La negación dentro del discurso del cambio climático del barbarismo, que también caracteriza a la humanidad, es una forma clásica de represión traumática. Según Blanchot, el desafío fundamental consiste en elegir entre un futuro apocalíptico, al que se avanza a toda velocidad precisamente por la ausencia de la humanidad, o la construcción de una humanidad que posteriormente cambie el rumbo del futuro hacia una dirección más benigna. Por lo tanto, la cuestión no es asegurar el futuro de una humanidad inexistente, tal como la conocemos, sino crear una humanidad en primer lugar. Alenka Zupančič insiste en que:

(...) Blanchot no dice que como no hay un mundo (comunal) real no importa si el mundo se destruyera; es decir, no es que cínicamente dice: "iQue todo se vaya al infierno, de todos modos, el mundo tal como es no vale la pena!” Por el contrario, Blanchot sugiere que ahora que al menos tenemos una idea abstracta del mundo (la humanidad) en su conjunto, el mundo vale la pena más que nunca (Zupančič, 2018:19).

De hecho, la fantasmagoría de una humanidad global abstracta y, sin embargo, amenazada constituye un imaginario importante de la posverdad que se filtra en el discurso climático dominante. Al hacerlo, lo Real de los antagonismos de clase, y de otros antagonismos que atraviesan a la humanidad, se considera irrelevante $o$ al menos inferior. La negación fetichista, el rechazo a los antagonismos que forman la matriz de lo social, incluyendo los de clase, asegura que nada realmente cambiará. Transitar la fantasía de la posibilidad de una transición climática justa por medio de ajustes tecno-gerenciales y de modificaciones consumistas (neo)liberales requiere reconocer el trauma de la inexistencia de humanidad. También requiere que se reconozca que es precisamente esta no-existencia, esto es, no reconocer la dimensión de clase y otras dimensiones que atraviesan a la humanidad, lo que ya ha provocado la catástrofe climática para muchos. Transitar la fantasía requiere invertir el argumento dominante: para ello se debe reconocer que ya es demasiado tarde -ya sucedió el apocalipsis- y lo único que queda por hacer es embarcarse en un proceso de construcción de una verdadera humanidad, de crear en el mundo un mundo humano. Como dijo The Invisible Committee:

Es inútil esperar por un gran avance, por la revolución, por el apocalipsis nuclear o por un movimiento social. Seguir esperando es una locura. La catástrofe no está llegando, está acá. Ya estamos al interior del colapso de una civilización. Esta es la realidad dentro de la cual debemos tomar partido (The Invisible Committee, 2009:138). 
ERIK SWYNGEDOUW

Esto último requiere de una politización radical en primer plano. $\mathrm{O}$, en otras palabras, si realmente queremos tomar en serio la problemática ecológica, tenemos que correr el tema de la ecología al terreno de la politización agonística motivada por una continua lealtad a lo que Alain Badiou llama la pasión por la posibilidad real y la necesidad de un mundo común igualitario. Es por medio de un proyecto político como éste que podría darse lugar a un clima común y propicio. 


\section{Q Bibliografía}

» Anderson, K. (2019). Nature Cannot be Fooled. Recuperado de: https://reddmonitor.org/2019/09/05/nature-cannot-be-fooled-kevin-anderson-on-mitigationas-if-climate-mattered/

» Andreucci, D. (2019). Populism, Emancipation, and Environmental Governance: Insights from Bolivia. Annals of the American Association of Geographers, 109(2), 624-633.

» Badiou, A. (2007). De Quoi Sarkozy est-il le Nom? París: Nouvelles Éditions Lignes.

» Beckett, S. (1958). Endgame. Nueva York: Grove Press. (Publicación original: 1957).

» Blanchot, M. (1971). l’Apocalypse Deçoit. En L'Amitié (pp. 118-127). París: Gallimard.

» Calder, W.E. (2011). Combined and Uneven Apocalypse. Washington, D.C.: Zero Books.

»Canovan, M. (1999). Trust the People! Populism and the Two Faces of Democracy. Political Studies, 47, 2-16.

» Dean, M.M. (1999). Governmentality: Power and Rule in Modern Society. Londres: Sage.

» Dodds, J. (2011). Psychoanalysis and Ecology at the Edge of Chaos. Londres: Routledge.

» Ernstson, H. y Swyngedouw, E. (2018). Interrupting the Anthropo-obScene: Immuno-biopolitics and Depoliticising Ontologies in the Anthropocene. Theory, Culture, Society, 35(6), 3-30.

»Esposito, R. (2008). Bios: Biopolitics and Philosophy. Minneapolis: University of Minnesota Press.

》Esposito, R. (2011). Immunitas. Cambridge: Polity Press.

» Esposito, R. (2012). Terms of the Political: Community, Immunity, Biopolitics. Nueva York: Forsham University Press.

» Feltham, O. (2008). Live Badiou. En Live Theory (pp. 136-139). Londres: Continuum.

» Fink, B. (1995). The Lacanian Subject: Between Language and Jouissance. Princeton: Princeton University Press.

» Fletcher, R. (2013). How I learned to stop worrying and love the market: virtualism, disavowal, and public secrecy in neoliberal environmental conservation. Environment and Planning D: Society and Space, 31, 796-812.

» Fletcher, R. (2018). Beyond the End of the World: Breaking Attachment to a Dying Planet. En I. Kapoor (Ed.), Psychoanalysis and the Global (pp. 48-69). Londres: University of Nebraska Press.

» Garcia, P.O. (2015). Sous l'Adaptation, I'Immunité. Etude sur le Discours de I'Adaptation au Changement Climatique. Tesis doctoral en Geografía, Universidad Grenoble Alpes. 
» Gore, A. (Guión) (2006). An Inconvenient Truth. [Película]. Lawrence Bender Productions.

》 Harper, E.T. (2020). Ecological Gentrification in Response to Apocalyptic Narratives of Climate Change: The Production of an Immuno-political Fantasy. International Journal of Urban and Regional Research, 44(1), 55-71.

》 Harvey, D. (2019). Marx, Capital and the Madness of Economic Reason. Londres: Profile Books.

》 Hoggett, P. (2013). Climate Chante in a Perverse Culture. En S. Weintrobe (Ed.), Engaging with Climate Change. Psychoanalytic and Interdisciplinary Perspectives (pp. 56-71). Londres: Routledge.

» Hulme, M. (2008). Geographical Work at the Boundaries of Climate Change. Transactions of the Institute of British Geographers, 33, 5-11.

" Klein, N. (2014). This Changes Everything: Capitalism vs. the Climate. Londres: Penguin.

» Lacan, J. (2007). The Seminar of Jacques Lacan Book XVII. The Other Side of Psychoanalysis. Nueva York: W.W. Norton.

» Lacan, J. (2014). The Seminar of Jacques Lacan: Anxiety (Vol. 10). Cambridge: Polity Press.

» Laclau, E. (2005). On Populist Reason. Londres: Verso.

» Latour, B. (2004). Why Has Critique Run out of Steam? From Matters of Fact to Matters of Concern. Critical Inquiry, 30 (Winter), 225-248.

» Mannoni, O. (1969). Clefs pour l'imaginaire ou l'Autre Scène. París: Editions du Seuil.

» Mannoni, O. (2003). I Know Well, But All the Same... En M. Rothenberg, D. Foster y S. Žižek (Eds.), Perversion and the Social Relation (pp. 68-92). Durham: Duke University Press.

» Mbembe, A. (2003). Necropolitics. Public Culture, 15(1), 11-40.

" McCarthy, J. (2019). Authoritarianism, Populism, and the Environment: Comparative Experiences, Insights, and Perspectives. Annals of the American Association of Geographers, 109(1), 301-313.

》 Miller, T. (2017). Storming the Wall: Climate Change, Migration, and Homeland Security. San Francisco: City Lights Books.

» Mudde, C. (2004). The Populst Zetigeist. Government and Opposition, 39, 542-563.

»New, M., Liverman, D., Schroder, J. y Anderson, K.L. (2011). Four Degrees and Beyond: The Potential for a Global Temperature Increase of Four Degrees and its Implications. Philosophical Transactions of the Royal Society A: Mathematical Physical and Engineering Sciences, 369, 6-19.

» Neyrat F. (2014). Critique du Géo-Constructivisme: Anthropocène et Géoingénierie. Multitude, 56, 37-47.

» Neyrat F. (2019). The Unconstructable Earth. An Ecology of Separation. Nueva York: Fordham University Press.

» Neyrat, F. (2010). The birth of Immunopolitics. Parrhesia, 10, 31-38.

» Observatorio Mauna Loa (2021, 5 de octubre). Monthly Average Mauna Loa $\mathrm{CO}_{2}$. 
Trends in Atmospheric Carbon Dioxide. Global Monitoring Laboratory. Recuperado de: https://gml.noaa.gov/ccgg/trends/mlo.html

"Parenti, C. (2011). Tropic of Chaos: Climate Change and the New Geography of Violence. Nueva York: Bold Type Books.

"Pellizoni, L. (2015). Ontological Politics in a Disposable world: The New Mastery of Nature. Farnham: Ashgate.

》Pohl, L. y Swyngedouw, E. (2021). What Does Not Work: The Spectre of Lacan in Post-Foundational Political Thought. En F. Landau, L. Pohl y N. Roskamm (Eds.), (Un)Grounding: Post-Foundational Geographies. Berlín: [TRANSCRIPT]. En prensa.

» Rancière, J. (1998). Disagreement. Minneapolis: University of Minnesota Press.

"Smith, N. (2008). Uneven Development: Nature, Capital and the Production of Space (3ra ed.). Londres: University of Atlanta Press.

"Swyngedouw, E. (2005). Governance Innovation and the Citizen: The Janus Face of Governance-beyond-the-state. Urban Studies, 42(11), 1-16.

"Swyngedouw, E. (2007). Impossible/Undesirable Sustainability and the PostPolitical Condition. En J.R. Krueger y D. Gibbs (Eds.), The Sustainable Development Paradox (pp. 13-40). Nueva York: Guilford.

》Swyngedouw, E. (2010a). Apocalypse Forever? Post-Political Populism and the Spectre of Climate Change. Theory, Culture, Society, 27(2-3), 213-232.

》Swyngedouw, E. (2010b). Trouble with Nature. Ecology as the New Opium for the People. En J. Hillier y P. Healey (Eds.), Conceptual Challenges for Planning Theory (pp. 299-320). Farnham: Ashgate.

»Swyngedouw, E. (2018a). $\mathrm{CO}_{2}$ as Neo-liberal Fetish - The Love of Crisis and the Depoliticized Immuno-Biopolitics of Climate Change Governance. En D. Cahill, M. Cooper, M. Konings y D. Primrose (Eds.), The SAGE Handbook of Neoliberalism (pp. 295-306). Londres: Sage.

"Swyngedouw, E. (2018b). Promises of the Political: Insurgent Cities in a PostDemocratic Environment. Cambridge: MIT Press.

» The Invisible Committee (2009). The Coming Insurrection. Cambridge, MA: The MIT Press.

》 The Royal Society (2009). Geoengineering the Climate: Science, Governance and Uncertainty. Londres: The Royal Society.

»Weintrobe, S. (2013). The Difficult Problem of Anxiety in Thinking about Climate Change. En S. Weintrobe, Engaging with Climate Change - Psychoanalytic and Interdisciplinary Perspectives (pp. 33-47). Londres: Routledge.

»Žižek, S. (1989). The sublime object of ideology. Londres: Verso.

» Žižek, S. (1997). The Plague of Fantasies. Londres: Verso.

» Žižek, S. (2006). Against the Populist Temptation. Critical Inquiry, 32(Spring), 551574.

» Žižek, S. (2017). Incontinence of the Void: Economico-Philosophical Spandrls. Cambridge: The MIT-Press.

»Zupančič, A. (2018). The Apocalypse is (Still) Disappointing. S: Journal of the Circle for Lacanian Ideology Critique, 10-11, 16-30. 
ERIK SWYNGEDOUW

\section{Erik Swyngedouw / erik.swyngedouw@manchester.ac.uk}

Profesor de Geografía en la Universidad de Manchester. Sus intereses de investigación incluyen la Ecología Política Urbana, el conflicto hidrosocial, la gobernanza urbana, la democracia y el poder político, la política ambiental y la política de la globalización. Anteriormente fue profesor de Geografía de la Universidad de Oxford y ocupó la Cátedra Vincent Wright en Science Po, París (2014). Ha obtenido el doctorado honorario de la Universidad de Roskilde en Dinamarca y de la Universidad de Malmö en Suecia. Entre sus publicaciones se pueden mencionar: Social Power and the Urbanization of Water (OUP, 2004), Liquid Power (MIT, 2015), Promises of the Political (MIT, 2018), The Post-Political and its Discontents (co-editado con Japhy Wilson, Edinburgh University Press, 2014) y Urban Political Ecology in the Anthropo-obscene (coeditado con Henrik Ernstson, Routledge, 2018). 